\title{
LOS CONDENADOS DI BENITO PÉREZ GALDÓS: DELL'ONORE E DEL RISCATTO
}

\author{
Lorenzo Spurio \\ Poeta, scrittore, Roma
}

https://doi.org/10.33676/EMUI_nomads.61.07

Cuando no hay justicia, nosotros, el pueblo, la inventamos ${ }^{1}$. (47)

Nei fruttuosi studi letterari in ambito spagnolo in merito alla tematica ampiamente trattata dell'onore coniugale nella produzione drammaturgica merita senz'altro una trattazione anche Benito Pérez Galdós (1843-1920). L'autore spagnolo, originario delle isole Canarie, mancato vincitore del Premio Nobel nel 1912 per il suo accecante anticlericalismo, rimane per lo più ricordato per la sua corposa produzione narrativa, per la sequela dei romanzi storici del progetto denominato Episodios nacionales, in cinque serie, prima e per la sua novela realista poi con particolare attenzione a Fortunata y Jacinta, forse la sua opera maggiore.

Significativa risulta anche la produzione teatrale di Galdós che, nel periodo compreso tra il 1861 e il 1921, vide la scrittura e la rappresentazione di circa una ventina di opera. L'ultima composta, in ordine di tempo, Antón Caballero (1920) rimase incompiuta a causa della sua malattia (era diventato cieco nel 1919) e la sopravvenuta morte; essa venne data a conoscere in forma incompleta l'anno successivo.

La fortuna teatrale di Galdós, sebbene alcune delle sue opere ottennero discreto successo, fu per lo più adombrata dalla sua produzione narrativa dove eccelse. In uno dei più completi manuali di letteratura spagnoli circolati per prima in ambito accademico nel nostro Paese si legge: «Per quanto fosse senz' altro un ottimo autore teatrale, tuttavia la sua fama di romanziere superò qualsiasi altra»»². "l critico José García López ha osservato che «Galdós poseía un gran instinto dramático, pero le faltaba el dominio de los recursos de la escena. Por eso sus obras, aunque dotadas de verdadero interérs humano, resulta lentas, poco ágilesı ${ }^{3}$.

L'opera della quale vorrei parlare è Los condenados del 18944. Essa offre una serie di elementi per porre l'attenzione sulla società spagnola dell'epoca ma anche per connettere l'opera specifica a una serie di altre realtà romanzate e oggetto di

\footnotetext{
I Battuta di Barbués che rivela una moda assai generalizzata e diffusa all'epoca: la giustizia sociale, sentita come veramente lontana e lenta, spesso difficilmente applicabile nel caso di banditi sempre in fuga e dunque imprendibili per le loro malefatte, veniva spesso risolta con sistemi di giustizia personale, di vendetta trasversale.

2 Cento autori spagnoli, Pubblicazioni del Seminario di Spagnolo, Università Commerciale "Luigi Bocconi", La Goliardica, Milano, 1957, p. 430.

3 José GARCía LóPEz, Historia de la literatura española, Vicens Vives, Barcelona, 2006, p. 568.

${ }^{4}$ L'anno si riferisce allo estreno (debutto) dell'opera mentre la pubblicazione in volume avvenne l'anno successivo.
} 
produzioni teatrali del periodo attorno ai temi della famiglia, del matrimonio, dell'adulterio e della strenua difesa dell'onore. Temi, questi, che nel dramma rurale di Jacinto Benavente e di Eduardo Marquina trovarono massima espressione (secondi, forse, solo alla Teresa di Leopoldo Alas alias Clarín, autore del quale Galdós fu molto amico) rispettivamente con le opere La malquerida (1913) e La ermita, la fuente y el río (1927). Sulla medesima linea va ricordata la centralità del concetto di honra nella trilogía dramática di Federico García Lorca che fu proprio "pupillo" di Marquina e che con La casa de Bernarda Alba completò, pochi mesi prima di venire fucilato, quella "narrazione per quadri" come un documental fotográfico, della terra spagnola, in particolare dell'Andalusia, capace di assurgere a "metafora" della provincia spagnola.

Un genere, quello del dramma rurale, iniziato in terra catalana con le opere di José Feliú y Codina, divenuto tipico e tipicizzante della letteratura spagnola (in Italia qualcosa di simile, di paragonabile può dirsi La figlia di lorio di Gabriele D'Annunzio considerato però come "dramma pastorale" e i romanzi di Grazia Deledda tra i quali Canne al vento, Elia Portolou e altri) che avrebbe contraddistinto - con esiti ed echi differenti - anche la produzione dei fratelli Quinteros sino ad Alejandro Cajona con Las tres perfectas casadas (1941) e La dama del alba (1944).

Los condenados è un'opera teatrale che si compone di tre atti e un prologo che venne messa in scena per la prima volta a Madrid presso il Teatro de la Comedia il 11 dicembre 1894 con Carmen Cobeña, Emilio Thullier e Miguel Cepillo nei ruoli principali (rispettivamente di Salomé, José e Santiago). Come ricordano le cronache del periodo e la poca critica raggiungibile attraverso la rete, l'opera non ottenne il successo sperato dall'autore. L'esito fu alquanto deludente, come ricorda anche Gloria López Forcén che nel suo saggio mette in evidenza come il prologo inserito da Galdós, in seguito al fracaso de la obra, era mosso da far chiarezza su quelli che la critica aveva recepito come punti di debolezza dell'opera da decretarne l'infelice esito. Riporto alcuni estratti significativi del prologo in cui non può non percepirsi un certo fastidio dell'autore dinanzi il mancato recepimento della sua opera: "Las obras de uno y otro género, así las muy pensadas y con cariño escritas, como las compuestas a vuela pluma, no son más que la mitad de una proposición lógica, y carecen de sentido hasta que no se ajustan con la otra mitad, o sea el público. ¿̇Casa? Resulta el conjunto verdad, el éxito. ¿̀No casa? Pues de seguro hay error grave en una de las partes, o en las dos. [...] El fin de toda obra dramática es interesar y conmover al auditorio, encadenando su atención, apegándole al asunto y a los caracteres, de suerte que se establezca perfecta fusión entre la vida real, contenida en la mente del público, y la imaginaria que los actores expresan en la escena. Si este fin se realiza, el público se identifica con la obra, se la asimila, acaba por apropiársela, y es al fin el autor mismo recreándose en su obra. El drama Los condenados no produjo en el público, al menos en la ocasión de su estreno, el efecto a que aspira toda obra de teatro» (3) ${ }^{5}$. Lucido nel rivelare il «desgraciado éxito» (5) Galdós decise d'inserire questo lungo apparato argomentativo in apertura all'opera con l'intento di dare alcune possibili piste

\footnotetext{
5 Tutte le citazioni dall'opera sono tratte dalla sua versione digitale: BenITO PÉrez GaLdós, Los condenados, Freeditorial, opera presente in rete in formato PDF liberamente scaricabile.
} 
d'indirizzo nel corso della lettura6: se il pubblico al debutto lo aveva in qualche modo "cassato" (lui stesso lo aveva definito «en parte distraído, en parte hostil», 5), quale probabilità ci sarebbe stata che l'opera venisse presa in considerazione una volta in folio?

Prima di avvicinarci all'argomento di questo drama mi sembra utile poter investigare, nei limiti del consentito, ancora meglio questo aspetto del fallimento dell'opera in oggetto e, in generale, della debolezza-a fronte della produzione in prosa - dell'opera drammaturgica di Galdós: «El mérito de la producción dramática de Galdós está más que en lo teatral, en lo psicológico; [...] viene a ser una reacción contra el furibondo neorromaticismo de Echegaray. Es el suyo un teatro a base, no de gestos estridentes, sino de pasiones y caracteres auténticos; un teatro realista, aunque matizado de elementos simbólicos, y en quel lo sobrenatural tiene también su papelı7 ha sostenuto José García López.

Gloria López Forcén, professoressa presso l'Università di Barcellona, nel suo mirabile saggio ben evidenzia quelli che possono essere le ragioni riconducibili al mancato successo dell'opera analizzando con perizia non solo le caratteristiche tecniche dell'opera ma anche la macchina organizzativa dietro alla messa in scena e un aspetto considerato non di poco conto ovvero, come vedremo dalla citazione che segue, che Galdós scrisse l'opera pensandola per essere interpretata proprio da un' attrice (la madrilena María Guerrero, una delle maggiori del teatro dell'epoca), cucendola alle sue peculiarità come un vestito e che, nel momento che per ragioni di carattere personale questa non poté acutar, allora l'opera fu sottoposta a necessarie misure correttive, rivisitazioni e modifiche: «Posiblemente Los condenados no sea una de las mejores obras galdosianas, sin embargo no es, sin duda, la peor y la perplejidad con que Galdos vivió la crítica negativa nos habla de una confianza en ella. Los factores externos a la obra en sí, - el cambio de primera actriz, los retoques de última horo, las expectativas creadas en el público al ser una obra para "la Guerrero" ayudaron a que un texto novedoso, no tuviera si no el éxito, por lo menos el interés que sin duda se merece y que hoy todavía podemos apreciar. La creación de unos personajes complejos, poco previsibles, actores de sus proprias vidas más allá de los peligros a los que tal hecho les conduzca, ofrecen al lector de hoy un cierto deseo de verlas hechos "personas" no sé si en la tarima de un teatro o en la oscuridad e una sala de proyecciónı8.

A documentarci il fallimentare esito dell'opera di Galdós, oltre al prologo all'opera scritto in un secondo momento, sono alcune notizie che vennero diffuse sulla stampa l'indomani dell'estreno: «Mandada retirar esta ombra por su autor, visto que no logró despertar el interés del público, no hemos de encontrar en su crítica. Las obras teatrales se escriben, como los procesos, para que un Jurado, que es el público, dicte un fallo inmediato, y erróneo o acertado, justo e injusto, no cabe apelación: el drama o el proceso se archivan, y así quedan, para que un

\footnotetext{
6 "El fracaso del estreno de la obra lleva a Galdos la necesidad de explicar la obra por un lado, de profundizar en sus ideas sobre el teatro por otro, y de arrancarse, con un poquito de mal genio y con mucha razón, las espinas clavadas por la critica», in GLORIA LÓPEZ FORCÉN, "A próposito de un fracaso galdosiano: Los condenados", «Espéculo. Revista de estudios literarios», n¹6, Universidad Complutense de Madrid, Madrid, 2000.

7 José GARCía LóPEZ, Historia de la literatura española, op. cit., p. 568.

8 GLORIA LÓPEZ FORCÉN, "A próposito de un fracaso galdosiano: Los condenados", op. cit.
} 
jurisconsulto o un critico exija ante el derecho o ante la literatura en épocas muy posteriores una responsabilidad sin efecto material»"; "Esta obra drámatica del eminente novelista, no ha obtenido el éxito que La de San Quintín y Realidad, y ni aun el discutido de La loca de la Casa. [...] Dícese que su obra es verdaderamente simbolista» 10; A la veces diríase que Los condenados no es otra cosa que el delirar de una imaginación calenturienta. [...] Nada hay en la obra del Sr. Pérez Galdós que sea real, nada que nos impresione por extraordinario y hermoso. [...] Cuanto sucede en la escena no nos produzca emoción alguna; [...] a poco de levantada la cortina empiece a caer sobre la escena lo que un autor francés llama lluvia gris de la indiferencia y el fastidios ${ }^{1}$.

La vicenda del drama si sviluppa in un piccolo centro della valle di Ansó, nella zona di Huesca, e ha a che vedere con una storia di foschi legami che viene alla luce nel momento in cui Salomé, nipote più giovane del signore locale Gastón, viene promessa in sposa a un vomo di alto lignaggio, certo Santiago Paternoy che la ragazza, però, non ama. Vari altri personaggi intervengono nel corso dell'opera che si sviluppa in tre drammi.

Merita particolare attenzione il personaggio di Santamona, una vecchia saggia molto religiosa da essere considerata la "santita del pueblo» spesso intenta alla raccolta di erbe officinali ${ }^{12}$ che propugna alle persone in base ai loro vizi quale cura. Si tratta di un personaggio centrale dal momento che, come spesso accade in opere che hanno una dimensione rurale come questa, attua come consigliera ma anche come sorta di divinatrice di ciò che di lì a poco si appresta ad accadere. Le sue parole e i suoi consigli sono sempre tenuti in grande considerazione e addirittura, proprio per le sue proprietà taumaturgiche e divinatorie, c'è chi la teme con la conclusione che tutti hanno profonda reverenza e rispetto della donna, ritenuta quasi una sorta di medium tra il reale e la nebulosa di fatti - spesso marcati da un certo fatalismo - che s'imprimono a dettare le esistenze di tutti.

Barbués, definito nello schema dei personaggi quale «ansotano pudente» è uno dei proprietari terrieri locali che, di contro a Santamona che cerca sempre di favorire il bene, s'inserisce nella storia a più altezze con recriminazioni e insinuazioni, aizzando alla rottura tra le parti, inveendo e attuando in maniera rancorosa. Si capisce subito il perché quando viene chiarito che suo fratello è stato assassinato da un vomo.

La rete dei personaggi che si costruisce presenta le seguenti caratteristiche: la giovane Salomé - come abbiamo accennato - è promessa in sposa a Santiago Paternoy, signore honrado che ha il doppio della sua età ma Salomé non lo ama e non vorrebbe quell'unione. A motivare il suo disinteresse verso Salomé, impostole dallo zio Gastón, è il suo amore e legame clandestino con un certo José León, un bandito fuggitivo sotto falso nome che in passato ha condotto una vita immorale e peccaminosa e che ora, avvicinatosi alla bella Salomé, cerca di rigenerarsi e di darsi a una nuova vita. Barbués mette subito in chiaro le cose quando, sin dal primo

\footnotetext{
9 «El Día», miércoles 12 de diciembre de 1894

10 «El Correo Militan», miércoles 12 de diciembre de 1894

11 "La Correspondencia de España», miércoles 12 de diciembre de 1894

12 Nelle primissime battute il personaggio di Feliciana la descrive così: "Satamona, que tiene la manía de recoger en el monte ramos de hierbas aromáticas para adornar las habitaciones... y ahuyentar los malos pensamientos» (15). Poco dopo José León si riferirà a lei come «la ancianita que corta ramos en el montel.
} 
atto, fa osservare a Paternoy che la donna non lo ama e, soprattutto, che esistono delle voci in merito a Salomé ("Usted habrá oído ciertos rumores....), 17; "ahora, cuando sube al monte, en evez de coger avellanas maduras, coge hombres verdesı, 24). Tuttavia Barbués è il genere di persona che insinua ma poi non dichiara la realtà che conosce, avanza per illazioni contribuendo ad alimentare il discredito della persona, a macchiarne l'onore per cose che lui conosce ma che, per non compromettersi troppo, non intende rivelare con tanta facilità. Questa è l'impressione che si ha di lui, conoscitore di fatti che, se venissero svelati, decreterebbero da subito ta rottura dello schema e, forse, determinerebbero anche una vera tragedia: «Rumores de deshonra han llegado a estos oídos... Estos ojos, algo vieron también, no diré dónde ni cuándo, en la noche obscura» (24). A motivo di questo comportamento non è tanto un'invidia verso Paternoy (Barbués non è intenzionato ad avere Salomé) ma un l'odio che nutre nei confronti di José León animato da una venganza de la sangre: "Sospecho que ese hombre [José León] tuvo arte y parte en la muerte de mi hermano Alonso [...] y el incendio de tus casas, de las casas de los Paternoys, en hechors (25).

A questo punto, durante un colloquio tra Santamona e Salomé la prima le consiglia di rivelarsi allo zio e di dire la verità: che non intende sposare Paternoy e che ama José León. $\mathrm{Ma}$ in realtà fa molto di più perché mette in guardia la ragazza da quell'vomo che, anche secondo lei è una canaglia (¡no tienes salvación hija mia!, 34). I tentavi di Santamona di animare nella ragazza una presa di coscienza che le permetta di rivelarsi o, al contrario, di allontanarsi da quell'amore pericoloso, sono vuoti dal momento che nelle scene immediatamente successive José León probabilmente alle strette - la convince di fuggire con lui («A las diez, te espero en el robledad», 37, le dice l'vomo). La desdicha di questo poveraccio è tale che mentre i due stanno confabulando e organizzandosi per scappare, vengono ascoltati da Barbués che li coglie sul fatto e subito entra in scena, richiamando l'intervento dello zio della giovane, Gastón.

BARBUÉs: ¡Les he sorprendido!... ¡Salomé aquí... sola con él! ¡Sin duda concertaban la escapatoria! (43)

GASTÓN: ¡Ladrón de mi honra! Si sales vivo de aquí, será para ir a la cárcel. (44)

Tra il clamore della scoperta appena avvenuta e il livore già consolidato di Gastón per l'onta subita con l'assassinio di un suo familiare, la vendetta si compie, provvedendo a richiamare l'attenzione di Gastón che, in quanto padrone di quella casa, dovrà decidere in merito alla questione:

JOSÉ LEÓN: [Salomé] será mi esposa.

GASTÓN: ¡Eso no... Cristo! Antes la vea ya muerta que en poder tuyo. ${ }^{13}$

13 Anche questo aspetto, come quello del facile ricorrere a sistemi di vendetta e di giustizia personale, in tale ambiente rurale era particolarmente diffuso. Per questioni d'onore, come insegna 
JOSÉ LEÓN: Salomé es libre, mayor de edad...

GASTÓN: Que no, digo. Primero la mato.

PATERNOY: Estas cuestiones no pueden resolverse así. (47)

A questo punto, dopo la veemenza adoperata da Gastón, succede una cosa strana. A lui spetterebbe la decisione in merito alla questione: come gestire la situazione e come destinare sua nipote deshonrada Salomé e, invece, lascia che sia lo stesso Paternoy a decidere in merito": "iSancionas con tu autoridad esta indigna ingratitud! (50). Paternoy si erge giudice della questione ma non dà sentenze immediate su come agire, difatti preferisce prima avere una conversazione con la sua promessa sposa Salomé la quale, stavolta senza reticenze, le confida di non amarlo e di essere stata in procinto di fuggire con l'vomo che realmente ama, José León.

PATERNOY: Salomé, como ves, tu familia no quiere que seas mujer de ese hombre. Ni lo quiere tampoco el pueblo en que has nacido. [...] Te propongo entrar como arrepentida, con clausura temporal, en el convento de la esclavitud de Berdún, que yo protejo, y he dotado ampliamente. (50)

Il tono mite di Paternoy sembra fuori luogo, dinanzi alla gravità di quanto la donna ha commesso e forse cela una macchinazione che il lettore verrà a conoscenza solo in seguito. Di fatti sembra di assistere a un continuo "scarica barile" di competenze e di acquisizione di decisioni: Gastón scarica su Partenoy e quest'ultimo fa in modo che sia la stessa Salomé a decidere del suo futuro, vincolandone, però, la scelta. Si crea un po' di confusione sulla scena, Gastón, che ha demandato ad altri il compito di prendere misure repressive, non si sente soddisfatto, la decisione di Partenoy non sembra provvedere a una buona riparazione ma, avendo lui stesso riconosciuto Partenoy come juez supremo, alla fine l'vomo honrado sentenzia così:

la produzione drammatica da Calderón de la Barca in poi, era auspicabile la difesa e la protezione dell'onore (e della castità della giovane, figlia o nipote) a dispetto della sua stessa vita. Al disonore che poteva giungere da condotte depravate o relazioni clandestine della donna era preferibile la morte della stessa. In La casa de Bernarda Alba di Federico García Lorca a un certo punto si sentono delle urla provenire dalla strada, subito viene detto che si tratta di una donna che sta per essere linciata dal popolo perché ha partorito segretamente il figlio avuto da una rapporto clandestino. Questo esempio - se ne potrebbero portare molti altri - testimonia come il crimine commesso dalla donna sia spesso facilmente risolto con il mezzo del dar la morte, anche al di fuori di una sentenza giudiziaria, giudizio che, invece, come Barbués ben dice in quest'opera, è il popolo a dare.

14 Può essere considerato un punto di debolezza di quest'opera poiché, se lo zio si mostra tanto furibondo dinanzi a ciò che è successo e potrebbe decidere di uccidere l'vomo (bandito rinomato), decretare la stessa fine per la nipote o chiuderla in un convento, lascia che sia Paternoy a decidere il da farsi, quasi lavandosene le mani. 
PATERNOY: ¿ Quién me contradice? (Con arogancia) ¿Hay alguien que se atreve a replicarme? (Con despotismo) ¡A casa todo el mundo! (En medio de un profundo silencio, empiezan a retirarse). Aquí no ha pasado nada. (52)

Con questa chiusa sibillina e infingarda che tende a minimizzare l'accaduto ("Qui non è successo niente") si chiude il primo atto dell'opera.

L'atto che segue subito ci informa che Salomé non ha accolto l'invito di Paternoy, difatti non si trova reclusa in un convento, ma vive felicemente in una casa fatiscente assieme a José León: ha deciso di seguire il suo cuore. Le loro condizioni sono disagiate e precarie e, se non intervenisse la buona Santamona di nascosto a portare loro del cibo, probabilmente non avrebbero di che vivere.

Solo a questa altezza Galdós permette di far luce sull'enigmatico personaggio di José León dipinto con fosche tinte nel primo atto come bandito, fuori legge, colpevole di crimini. Viene detto di essere stato, oltre che un malvivente, un donnaiolo seriale, di aver avuto molte donne e dunque si apre la questione se realmente possa dirsi infatuato di Salomé e sia sincero e rispettoso nei suoi riguardi. Egli stesso, rievocando il suo maldestro passato, rivela:

JOSÉ LEÓN: [...] No pensaba más que en satisfacer mis locos apetitos. Donde hubiera pendencias, desorden, aventuras, embriaguez, juego, mujeres, allí estaba yo [...] todo aquel pasado escandaloso me inspira vergüenza, repugnancia. (62)

Stando alle sue parole, José León, avendo compreso gli errori commessi nel passato è ora in grado di smarcarsi da quell'età disdicevole dove ha agito in maniera disinibita non restio dal provare vergogna. Le «viejas conquistas» amorose di una volta divengono allora, nel momento presente della situazione in cui è coinvolto amorosamente con Salomé, un "cruelísimo castigo». Con l'intenzione di poter garantire a quella che sarà la sua donna (nel frattempo l'anziano prefe che avrebbe dovuto sposarli è morto) una vita normale è intenzionato ad acquisire la granjilla che solo riallacciando i rapporti con una sua vecchia fiamma, certa Feliciana che già Salomé aveva percepito come una sorta di "nemica" e così si reca dalla donna. Parimenti Salomé, anche per mezzo delle numerose voci che corrono, comincia a dubitare dell'onestà dell'vomo, essendo venúta a conoscenza delle sue pregresse relazioni amorose e, quando José León viene visto da altri confabulare a solas con Feliciana e la voce si sparge, la giovane Salomé comincia realmente a sentirsi offesa e deshonrada.

SALOMÉ: [...] ¡Vergüenza, justicia! No le tengo lástima! (Transición brusca) ¡Sí, le tengo lástima, sí, sí...! ¡Le vendí! ¡Ay, ay, qué horrible amargura! ¡Y lo llevarán a la cárcel, al patíbulo!... jMoriremos los dos! (75)

Dinanzi alla collusione col male (l'essere donna di un bandito senza nome, colpevole di delitti), Salomé viene condotta in schiavitù (come avrebbe dovuto 
fare ben prima, dietro sua libera scelta e su consiglio di Paternoy, quando era ancora in tempo, evitando di fuggire col delinquente) in un convento.

SALOMÉ: ¡Sáquenme de aquí! ¡Tengo miedo!... Maldita mil veces esas ruinas; maldita esta casa en que creí de encontrar la felicidad!... ¡Al convento!... Quiero rezar... aquí no puedo... quiero salvar mi alma. ¡Llévame con Dios!... (77)

Ci si approssima alla conclusione di questo dramma imprevisto; nelle ultime scene vediamo Salomé, ormai internata nell' istituto religioso dove tenterà di espiare la sua colpa, che «viste traje monjil de educanda, con toca y rosario al cinto» (10). Durante l'ultimo colloquio che Salomé ha con José León la donna non fa che allontanarlo, avendo ben compreso il grave errore nel quale è caduta: "Vete. No me atormentes. Yo estoy muerta. Yo descanso mientras no mueras como yo no serás conmigo en paz. Tú estás vivo y cargado de culpası (104). Dinanzi ai peccati commessi il pensiero della donna è che solo con la morte saranno ripagate le onte e le violenze perpetuate dall'vomo: non potrà esserci in vita un sistema di epurazione dei crimini. L'idea, inoltre, che la donna si descriva come morta non solo vuol far riferimento alle condizioni privative della vita claustrale ma si coniuga a un sentimento di nebbia della coscienza che, come ha sottolineato la critica, la direzionerà velocemente a una confusione emotiva e intellettiva da relegarla a uno stadio d'insania. Qualcosa di analogo, questo sentire di essere morti ben prima che la morte arrivi, fa pensare al personaggio di Mariana Pineda, la liberale granadina nella celebre omonima opera di Federico García Lorca incarcerata da Pedrosa e presto condotta al patibolo. Fa pensare anche a Giovanna d'Arco, altra rivoluzionaria che, ormai divenuta pazza, è pervasa da allucinazioni, vede la sua persona sdoppiarsi ed è contraddistinta da un progressivo deterioramento mentale. Per quanto concerne Salomé si chiarisce anche la gravità della sua colpa commessa che non è tanto quella patente e nota a tutti dell'essersi accompagnata con un delinquente, di non aver accettato il promesso sposo e di essere fuggita di casa con il suo amato, ma di aver nutrito, nella sua intimità, un sentimento di profondo rancore nei confronti di Feliciana Bellindo - una delle tante conquiste di José León - difatti solo ora veniamo a sapere che aveva tentato di ucciderla nel sonno preferendo poi sottrarle i suoi due figli.

Comincia a scorrere per la via la processione religiosa che è aperta dalla madre superiora e alla quale prende parte un po' l'intero pueblo. José León - definito da Paternoy come uperverso» (72) - sembra in procinto - se non di chiedere apertamente scusa per le sue malefatte - per lo meno volerle confessare: «largo tiempo he vivido en el reino de la mentira, pero ya está cerca de aquí» (113). La confessione non tarda ad arrivare e l'vomo confessa l'uccisione di Alonso Barbués e di altre persone non identificabili nel dramma, furti commessi, incendi ad abitazioni, come quelli alla casa di Paternoy, crimini dai quali avrebbe voluto distanziarsi ed epurarsi proprio iniziando una nuova vita con Salomé la donna che con il suo casto e onesto amore avrebbe potuto riabilitarlo. 
JosÉ LEÓN: El hombre de que os hablo reconosce hoy públicamente sus abominaciones... y se entrega indefenso a la justicia humána... y a la misericordia divina. (114)

Dopo la confessione - che non corrisponde a un vero atto di pentimento - le ultime battute dei principali protagonisti sono le seguenti, prima della chiusura definitiva del telone:

PATERnOY (A José León): Ven a mí. Serás mi amigo, mi hermano.

José LEÓn (A Paternoy y a Santamona): Gracias nobles hijos de Ansó, espíritus de clemencia... Me debo a la expiación. Me sduce el suplicio; me enamora la muerte.

PAternoy (A Solomé, ansioso, pidiéndole su concurso para convencerle): Tú, háblale... disuádele de esa horrible idea del martirio.

SALOMÉ: ¿łY, yo sentencio ahora? (con iluminismo y acento mistico). Quiero que venga a mí... Le condeno a muerte...

José LEÓN: Vamos (presuroso se agarra a Barbués y corren ambos hacia la salida. Telón).

Una delle cose senz'altro sobresalientes del dramma è che, ancora una volta, si manifesti confusione tra giudicato e giudicante, tra colpevole e giudice, difatti, ancora una volta Paternoy tiene a sentire il parere di Salomé in merito al destino da dare al suo amato José León che ha appena confessato la sua condotta depravata. II fatto che chieda a Salomé come agire nei confronti di José León rappresenta al contempo un ulteriore atteggiamento crudele dell'vomo e una banalità dal momento che egli sa già come dovrà reagire e decidere in merito e che, di contro, sa che Salomé, orami divenuta pazza, non sarebbe più in grado di difendere quell'vomo. La decisione ultima di dar la morte a José León, professata dalla donna che l'aveva amato, non è che l'ennesimo stratagemma per servire, in maniera pedagogica dinanzi al popolo, una lezione di avvertimento e scongiura dinanzi a quel che accade nei confronti di chi se porta mal. José León sarà di lì a poco impiccato, ma ciò non viene detto, viene lasciato intuire, è stafa la critica ha scrivere quel finale che, pur tra i vari contorni sfumati, in un certo senso era pronosticabile nel corso dell'opera. La morte dell'vomo è l'esatta pena che viene data a un dissoluto dietro sua confessione. Avviene, pertanto, dopo un contradditorio più affine a uno stato egualitario di diritto che non ha una società arcaica dove, come ricordava Barbués, la giustizia viene fatta dagli vomini, dal paese. Difatti e l'estrema truce genialità di Paternoy che, nel dare ad altri il compito che in quel contesto è solo suo (giudicare, decidere, far eseguire la pena), più che boia finisce per apparire come emblema di giustizia, custode del benessere collettivo quando in realtà con il suo architettato comportamento non fa altro che attuare la vendetta che era andato costruendo.

Si è detto in apertura di questo saggio del fracaso dell'opera nel momento in cui si tenne il suo debutto nel 1894. L'interesse verso Los condenados venne riacceso, solo in parte, nel 1915 quando l'opera venne riproposta in una nuova versione con 
l'indicazione, a far seguito al titolo, di revisión literaria. In molti non apprezzarono il tentativo disperato di riproporre un'opera che la critica, da ogni lato, aveva profondamente bersagliato; il reestreno si tenne il 6 aprile 1915 per espressa volontà di Federico Oliver, direttore della Compañía del Teatro Español. Il maggior merito di questa realizzazione venne principalmente ascritto dall'interpretazione dell'attrice Carmen Cobeña nel ruolo principale di Salomé. Sebbene, come scrisse Antonio Soler sulla stampa l'indomani della rappresentazione del 1915, la Cobeña «con su exsquisitez de mujer de talento no ha querido que Galdós conserve en su memoria el recuerdo de la noche del estreno de su obra en la Comedia» 15 tuttavia è questo ciò che la critica sul teatro di Galdós ci ha consegnato: quella di un'opera debole, non convincente, profondamente attaccata dalla critica che rese insoddisfatto il pubblico e il cui esito, chiaramente, non poté che fastidiar il suo autore.

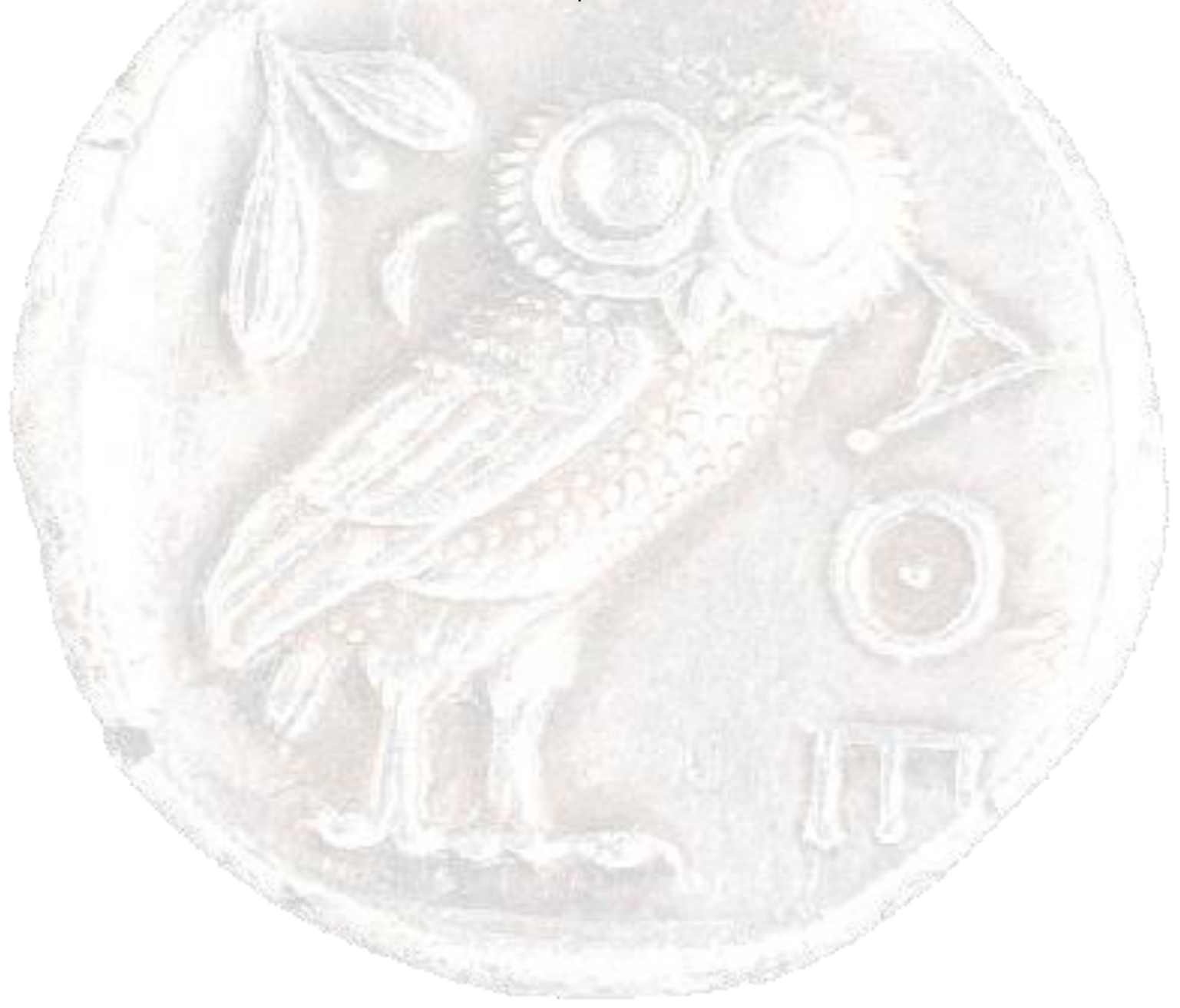

\footnotetext{
${ }^{15}$ E poi, ancora, nello stesso articolo, in riferimento all'attrice Cobeña, si può leggere: «Merced a ella, anoche estrenó Galdos su drama Los condenados, obteniendo en aquella su primera representación un éxito clamoroso, inmenso, indiscutible....l.
} 


\section{Bibliografia}

"Los condenados, drama en tres actos y en prosa de D. Benito Pérez Galdós", «El Día», miércoles 12 de diciembre de 1894.

"Los condenados, por D. Benito Pérez Galdós", "El Correo Militan», miércoles 12 de diciembre de 1894

"Reseña de Los condenados de Benito Pérez Galdós", "La Correspondencia de España», miércoles 12 de diciembre de 1894.

Cento autori spagnoli, Pubblicazioni del Seminario di Spagnolo, Università Commerciale "Luigi Bocconi", La Goliardica, Milano, 1957.

GARCía López José, Historia de la literatura española, Vicens Vives, Barcelona, 2006

https://webs.ucm.es/info/especulo/numerol6/galdos.html

LÓPEZ FORCÉn GLORIA, "A próposito de un fracaso galdosiano: Los condenados", "Espéculo. Revista de estudios literarios», $n^{\circ} 16$, Universidad Complutense de Madrid, Madrid, 2000, enalce:

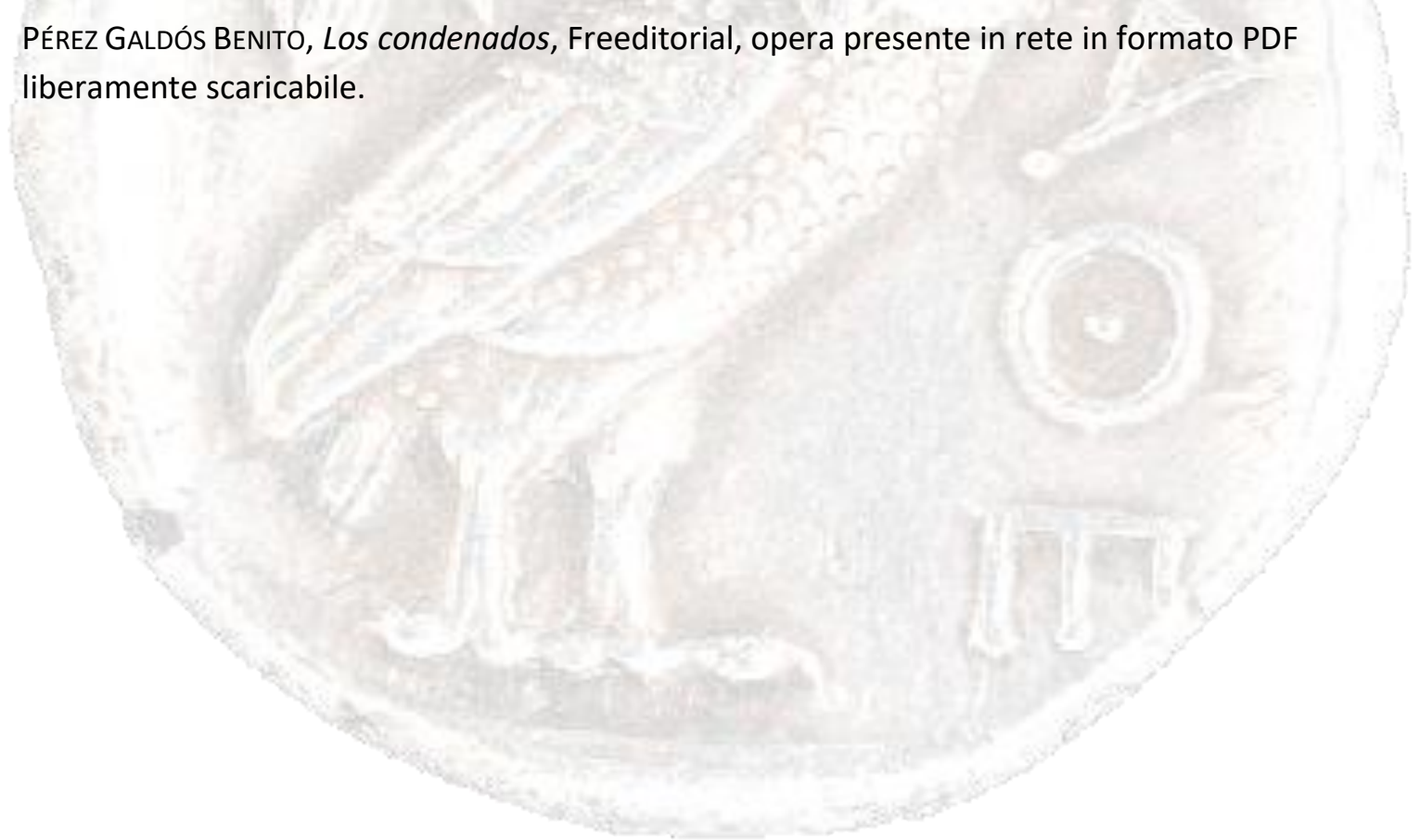

